$$
\begin{aligned}
& \text { CONF- } 960850--21 \\
& \text { ANL/MSD/CP - } 89215
\end{aligned}
$$

Effects of Vortex-Vortex Interactions on Ion-Track Pinning in High-T $T_{c}$ Superconductors

\author{
K.E. Gray, D.G. Steel, J.D. Hettinger, ${ }^{*}$ D.J. Miller, B.R. Washburn, \\ M. Ware, J.T. Parkman, M.E. Yoder, C. Moreau \\ Materials Science Division and the Science and Technology Center for Superconductivity \\ Argonne National Laboratory, Argonne, IL 60439
}

M.M. Eddy

Superconducting Technologies, Inc., Santa Barbara, CA 93111

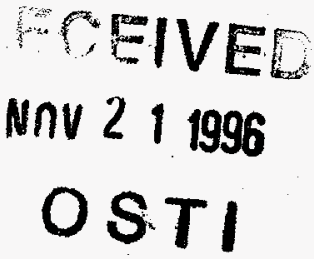

University of Chicago es Operalor of Anponne Netionel Laboratory (A Argonne") under Contract Na. W-31-103 ENG-38 with the U.S. Department of Energy. The U.S. Government retains for liself, and others acting on its behali, a paid-up, non exclusive, irrevocablo wortowice license in said arkice lo reproducs, prepare dervative works, distibuto coples to the pubic, and

1996 Applied Superconductivity Conference, August 25-30, 1996, Pittsburgh, PA, to appear in IEEE Transactions on Superconductivity, Spring 1997.

Work supported by U.S. Department of Energy, Division of Basic Energy Sciences-Materials Sciences, and Energy Efficiency and Renewable Energy, as part of a DOE program to develop electric power technology, under contract \#W-31-109-ENG-38 and by the National Science Foundation Office of Science and Technology Centers under contract DMR \#91-20000. BRW, MW, JTP, MEY and CM acknowledge support by Divison of Educational Programs at Argonne.

*Present address: Dept. of Chemistry and Physics, Rowan College of New Jersey, Glassobro, NJ 08028. 


\section{DISCLAIMER}

This report was prepared as an account of work sponsored by an agency of the United States Government. Neither the United States Government nor any agency thereof, nor any of their employees, makes any warranty, express or implied, or assumes any legal liability or responsibility for the accuracy, completeness, or usefulness of any information, apparatus, product, or process disclosed, or represents that its use would not infringe privately owned rights. Reference herein to any specific commercial product, process, or service by trade name, trademark, manufacturer, or otherwise does not necessarily constitute or imply its endorsement, recommendation, or favoring by the United States Government or any agency thereof. The views and opinions of authors expressed herein do not necessarily state or reflect those of the United States Government or any agency thereof. 


\section{DISCLAIMER}

Portions of this document may be illegible in electronic image products. Images are produced from the best available original document. 


\title{
Effects of Vortex-Vortex Interactions on Ion-Track Pinning in High-T Superconductors
}

\author{
K.E. Gray, D.G. Steel, J.D. Hettinger*, D.J. Miller, B.R. Washburn, M. Ware, J.T. Parkman, M.E. Yoder, C. Moreau \\ Materials Science Division and Science and Technology Center for Superconductivity \\ Argonne National Laboratory, Argonne, IL 60439 \\ M.M. Eddy, \\ Superconducting Technologies, Inc., Santa Barbara, CA 93111
}

\begin{abstract}
Many superconductor applications rely on the ability to pin vortex lattices. This ability depends on structural defects to pin individual vortices, but vortex-vortex interactions often play an important role in pinning the other vortices. Experimental progress on this complex problem can be made by introducing random arrays of welldefined pinning centers and studying the flux dynamics for a range of vortex densities (i.e., fields). The results of such studies in epitaxial $\mathrm{Tl}_{2} \mathrm{Ba}_{2} \mathrm{CaCu}_{2} \mathrm{O}_{\mathrm{y}}$ films containing ion tracks show the importance of vortex-vortex interactions. As an example, the effective pinning field of the defects rises to many times the ion-dose field for temperatures well below $T_{c}$.
\end{abstract}

\section{INTRODUCTION}

The principal effect of linear ion-damage tracks is well established. It provides extremely strong pinning centers for magnetic vortices, and because the tracks can be made continuous, their effectiveness is not diminished in regions of field and temperature where the vortices behave two dimensionally [1], e.g., $\mathrm{Bi}_{2} \mathrm{Sr}_{2} \mathrm{CaCu}_{2} \mathrm{O}_{\mathrm{x}}$ at $77 \mathrm{~K}$. However, because thermal energy scales are not so different from pinning and vortex-vortex interaction energies, the detailed picture is richer, and the defects may provide additional benefits to applications. It is the purpose of this paper to further explore such ideas by connecting incisive experimental results with simple models.

In particular, we show that the randomness of the defect positions washes out any sharp distinction between fields less than or greater than the dose field, $B_{\phi}=\Phi_{0} / d_{0}^{2}$ where $d_{0}$ is the average defect spacing and $\Phi_{0}$ is the flux quantum. Although the fundamental field scale is still related to the defect density, a temperature-dependent effective dose field $B_{\dot{\phi}}$ is found: its value is small just above $T_{c}$, but because of vortex-vortex interactions it rises to many times $B_{\phi}$ for $T<<T_{c}$. Comparing data with a simple model of randomness, one concludes that the vortex lattice will distort by a reasonable fraction $(\sim 0.25-0.33)$ of the intervortex spacing, $a_{0}$, to find favorable ion-core pinning sites.

\footnotetext{
Manuscript received August 26,1996.

This work is supported in part by the U.S. Department of Energy, Basic Energy Sciences-Materials Sciences, and Energy Efficiency and Renewable Energy, as part of a program to develop electric power technology, under Contract No. W-3i-109-ENG-38, and the National Science Foundation under Contract No. DMR 91-20000. BRW, MW, JTP, MEY and CM acknowledge support by Division of Educational Programs.

"Present address: Rowan College, Glassboro, NJ.
}

Another important issue addresses the dimensionality of the vortices: Are they three-dimensional (3D) vortex lines, or $2 \mathrm{D}$ pancake vortices residing independently in every bilayer of closely-spaced $\mathrm{Cu}-\mathrm{O}$ planes? It is generally agreed that in the most isotropic HTS cuprate, fully-oxygenated $\mathrm{YBa}_{2} \mathrm{Cu}_{3} \mathrm{O}_{7}$, the vortices are 3D over much of the superconducting phase. However, experiments and theory for the highly-anisotropic HTS, like $\mathrm{Bi}_{2} \mathrm{Sr}_{2} \mathrm{CaCu}_{2} \mathrm{O}_{\mathrm{x}}$ and $\mathrm{Tl}_{2} \mathrm{Ba}_{2} \mathrm{CaCu}_{2} \mathrm{O}_{\mathrm{x}}$, predict crossovers [1] or phase transitions [2,3], from $3 \mathrm{D}$ vortex lines at low fields and temperatures to 2D independent pancakes at higher fields. Linear ion tracks have been used extensively to study the 3D or 2D nature of vortices [4-8]. The high-energy ions amorphize the superconductor in fine $(\sim 10 \mathrm{~nm}$ diameter $)$ columns along their linear trajectory which provide very strong columnar flux pinning centers. As such, a strong dependence of pinning on the angle between the tracks and the magnetic field is expected for 3D vortices, but not for independent, strictly 2D pancakes. Thus, ion-irradiated samples of $\mathrm{YBa}_{2} \mathrm{Cu}_{3} \mathrm{O}_{7}$ have demonstrated [4] the 3D nature of its vortices, while initial measurements on $\mathrm{Bi}_{2} \mathrm{Sr}_{2} \mathrm{CaCu}_{2} \mathrm{O}_{x}$ were interpreted [5] to imply 2D pancakes. More recent work $[6,7]$ has concluded that the vortices in $\mathrm{Bi}_{2} \mathrm{Sr}_{2} \mathrm{CaCu}_{2} \mathrm{O}_{\mathrm{x}}$ show a finite interbilayer coupling (or line tension) and thus behave 3D. We report measurements showing such 3D behavior at fields and temperatures where thermal activation [1] and scaling [9] measurements imply that the vortices exhibit strongly $2 \mathrm{D}$ behavior. We address this contradiction directly and show how the apparent 3D behavior may be consistent with a $2 \mathrm{D}$ thermal-activation limit and that the scaling behavior [9] may be the result of very strong intrinsic pinning by the $\mathrm{Cu}-\mathrm{O}$ layers, rather than strictly uncoupled $2 \mathrm{D}$ vortices.

\section{SAMPLE FABRICATION AND IRRADIATION}

Films of phase pure $\mathrm{Tl}_{2} \mathrm{Ba}_{2} \mathrm{CaCu}_{2} \mathrm{O}_{\mathrm{x}}$ were made at Superconductor Technologies, Inc., using laser ablation with a post-deposition anneal: they showed rocking curves with a full-width at half maximum of $<0.4^{\circ}$. Such films display very reproducible superconducting properties, particularly in high magnetic fields where their irreversibility behavior is very nearly identical from sample to sample. Before irradiation, the $8000 \AA$-thick films were patterned into a series of four microbridges, each $50 \mathrm{~mm} \times 1 \mathrm{~mm}$. Two of the microbridges were shielded from the ion beam using a $\mathrm{Ta}$ 
shield, so that samples with exactly the same process history could be compared with and without irradiation. Transport data on all unirradiated (and on all similarly irradiated) sections were virtually identical.

The ion irradiation was performed at the Argonne Tandem Linac Accelerator System (ATLAS) facility at Argonne National Laboratory using $650 \mathrm{MeV}$ Xe or Sn ions. Based on TRIM calculations, the energy deposited by such ions falls below the threshold for continuous tracks only after a distance of about $20 \mu \mathrm{m}$, which is much greater than the film thickness $(0.8 \mu \mathrm{m})$. A uniform lateral distribution of ion tracks was ensured by scattering the ion beam through a $\mathrm{Au}$ foil at a distance of $\sim 1 \mathrm{~m}$ from the sample (a simple Rutherford scattering algorithm was used to determine the $\mathrm{Au}$ thickness needed to achieve a specific ion distribution at the target position). Scattering the beam using the Au foil did not introduce any spaying of the columnar defects, but it had the additional advantage that part of the beam could be scattered into a Si detector for an in-situ measure of the ion flux. The Si detector was calibrated versus a Faraday cup and the resulting distribution of the ion beam was confirmed using a Faraday cup behind various sized apertures.

The defects were confirmed to be amorphous and columnar using transmission electron microscopy on a companion sample irradiated at the same time. Samples were compared before and after irradiation in a magnetic field using standard four-probe dc transport measurements. Both linear response (limit of ohmic resistance at small current density, J) and the very non-ohmic, high-current regime were probed as a function of temperature, $T$, magnetic field, $B$, and ion dose.

\section{VORTEX DIMENSIONALITY}

Upon ion irradiation, thin films generally show a larger reduction in the transition temperature, $T_{c}$, in comparison to bulk, e.g., single crystals. This may be caused [10] by the creation of substrate defects which can create stress at the film interface, thereby suppressing $T_{c}$. This reduction in $T_{c}$ becomes quite significant at higher doses, $B_{\phi}$. Using the resistive midpoint, $T_{c}$ is suppressed by $\sim 0.7 \mathrm{~K}$ for $B_{\phi}=1 \mathrm{~T}$, while for $B_{\phi}=2 T$, the difference is $\sim 2 \mathrm{~K}$. For the lowest measurable resistances, these were 1.5 and $4.8 \mathrm{~K}$.

Typical resistance data are shown in Fig. 1 for a $\mathrm{Tl}_{2} \mathrm{Ba}_{2} \mathrm{CaCu}_{2} \mathrm{O}_{\mathrm{y}}$ ( $\mathrm{Tl}-2212$ ) epitaxial film as a function of angle between the applied field and the c-axis. These were taken at various magnetic fields (1-7 T), at a temperature of $80 \mathrm{~K}$ and in the small-current limit (i.e., the linear currentvoltage, $\mathrm{I}(\mathrm{V})$, response regime in which the Magnus (Lorentz) force plays a negligible role in depinning vortices). These data are for that part of the film which has been irradiated with ions approximately parallel to the $c$-axis $\left(T_{c}=102.2 \mathrm{~K}\right)$ at an average density of $B_{\phi}=2 \mathrm{~T}$. Comparable data on the unirradiated section $\left(T_{c}=104.2 \mathrm{~K}\right)$ are shown in Fig. 2 .

Qualitatively similar data are found at other temperatures, and virtually identical data are found on other films. Data are normalized by $R_{n}$, the high-temperature, normal-state value extrapolated to $T_{c}$, which changes slightly after irradiation.

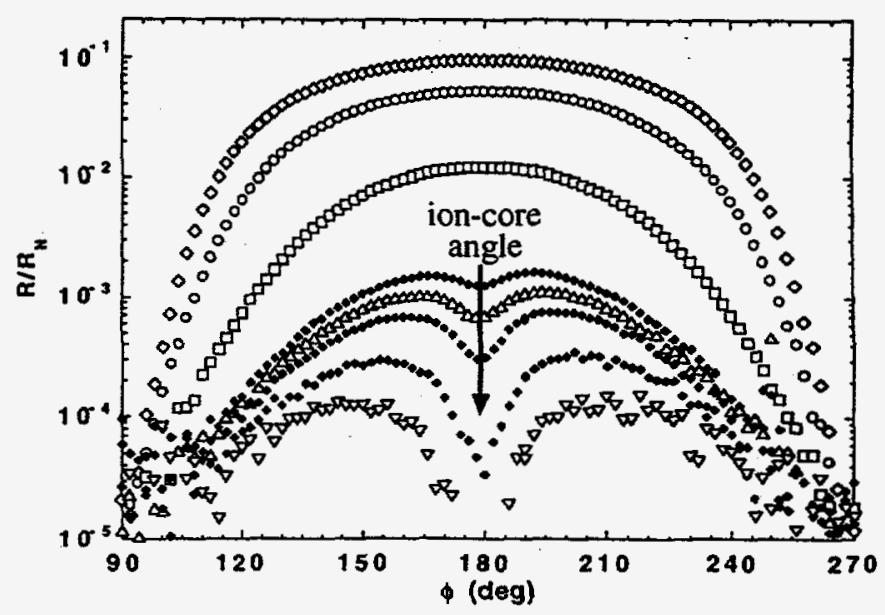

Fig 1. Normalized resistance vs. angle between c-axis and applied field for $B=1.5,1.7,1.9,2,2.1,3,5$ and 7 (top) $T$.

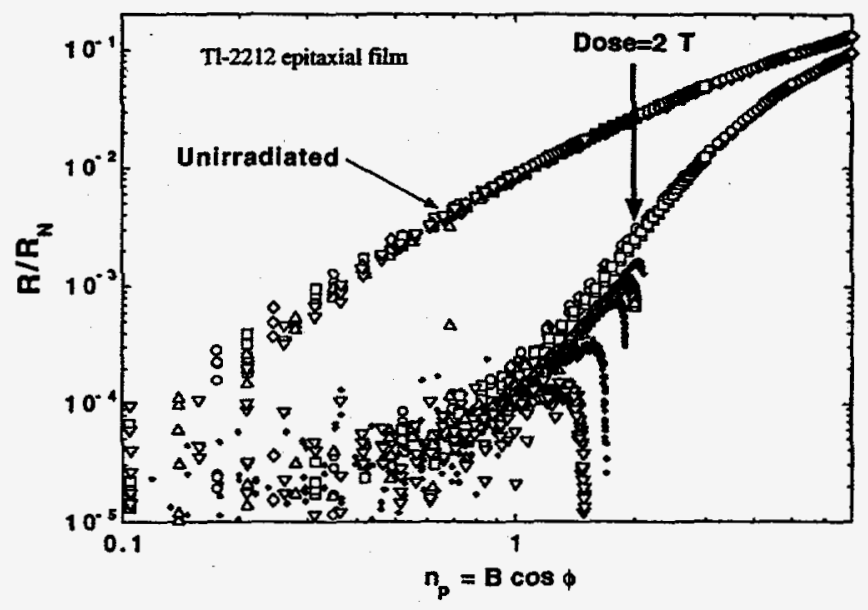

Fig. 2. Data of Fig. 1 with similar data on an unirradiated portion of film plotted against pancake vortex density. The dip of Fig. 1 appears as a downward spike here.

In the irradiated section, dip features are found with the field, c-axis and ion tracks all colinear (i.e., at $0^{\circ}$ and $180^{\circ}$ ), but only at the lowest fields. All the data of Fig. 1, plus the same full set of field values for the unirradiated section, are plotted in Fig. 2 as a function of $B|\cos \phi|$, which is the areal density of pancake vortices, $\mathrm{n}_{\phi}$, in the $\mathrm{Cu}-\mathrm{O}$ bilayers. In the unirradiated section (upper data sets), one observes essentially perfect scaling. Based on Ref. 9, such scaling behavior is often taken to be a sign of $2 D$ pancake vortices. The dissipation measured in the companion irradiated section (see Fig. 2) also scales at the highest fields (3-7 T), but a dip in dissipation is seen for fields aligned with the columnar ion tracks $(|\cos \phi|=1)$ at lower fields $(1.5-2.1 \mathrm{~T})$. These fields are well within both the scaling regime of the unirradiated films and the 2D regime of the thermal-activation model [1].

The strong dependence of pinning on the angle between the tracks and the field represented by the dip feature in Fig. 1 is expected for 3D line vortices, but not for independent 2D pancake vortices. Thus these results seemingly contradict 
both the thermally-activated 2D limit of [1] and the 2D limit implied by [9] from the scaling. We therefore discuss both cases in greater detail.

Thermally-Activated 2D Limit. Dissipation in linear response requires thermal activation, and in [1] this is only significant in the ' $2 \mathrm{D}$ limit', i.e., where $\mathrm{kB}_{\mathrm{B}} \mathrm{T}$ exceeds the coupling energy to adjacent bilayers. For the interbilayer coupling, a Josephson coupling energy, $E_{j}=e_{j} \Phi_{0} / B$, where $e_{j}$ is the intrinsic value per unit area is used and the area is determined by the magnetic length, $\sqrt{\Phi_{0} / B}$, where $\Phi_{0}$ is the flux quantum. The need to overcome the interbilayer coupling energy is based on the likely assumption that there is at least one very strong pinning center somewhere along the length of any vortex line. Experimental justification is presented in $[1,11]$. It should be emphasized that this model predicts strictly $2 D$ behavior only for $k_{B} T>E_{j}$. We thus consider the possibility that even in the dissipative mode with $\mathrm{k}_{\mathrm{B}} \mathrm{T}_{\sim} \mathrm{E}_{\mathrm{j}}$, that the dip in Fig. 1 can occur upon ion irradiation.

We specifically consider the lowest-energy configuration of pancake vortices in an irradiated sample (modeled by ignoring thermal fluctuations). Such a configuration exhibits stacks of pancake vortices within columnar tracks separated by 'unpinned' chains of pancake vortices, and is described in detail in [12]. It reproduces a dip for alignment, because then there are fewer 'unpinned' chains. If the above model [12] is applied to the lowest-field data of Fig. 1, the fits to the angular dependence are qualitatively reasonable, but the coupling strength compared to pinning is significantly larger than expected from other experiments or the enormous anisotropy of the material. The conclusion of [12] is that of vortexvortex interactions may be needed. Nonetheless, the qualitative results and discussion indicates that the apparent contradiction of the dip with [1] may be a semantical one: the line tension, or $E_{j}$, is never strictly zero (i.e., the system is never strictly 2D), and although its effect on thermal activation in linear response is small when $k_{B} T>E_{j}$, it still affects the number of pancake vortices that, on average, reside outside the columnar defects at a given field angle. A more complete model, which includes finite field effects and vortexvortex interactions, is clearly needed.

Two-Dimensional (2D) Scaling. In spite of the near perfect ' $2 \mathrm{D}$ ' scaling shown in Fig. 2 for the unirradiated section, it is clearly seen that the dissipation is not directly proportional to the pancake vortex density, $n_{\phi}=B|\cos \phi|$, although such behavior is approached at the highest fields. This is consistent with, and expected for, samples with a distribution of pinning energies. The shift in this distribution from the naturally occurring pinning sites to include the strong ion-defect pinning sites is also clearly visible in the scaled data for the irradiated films of Fig. 2 when compared with the unirradiated data: to achieve the same resistance in irradiated samples takes additional pancake vortices which are rigidly pinned in ion-defect pinning sites.

However, the apparent inconsistency between: the implication of 2D vortices because of such scaling; and the 3D-like dip feature of the irradiated films is still to be addressed. It should be pointed out that the scaling seen in Fig. 2 can also result from the very strong intrinsic pinning which prevents Josephson vortex segments (parallel to the ab- planes) from moving across such ab-planes. Then only the ab-plane component of the Lorentz force [13] can induce a time-average velocity, $v f f$, for the thermally-excited vortices. Note that $v_{f f}$ should be unaffected by the presence or absence of Josephson vortex segments because of the negligible dissipation (drag) associated with the small pair density between the bilayers. Finally, the Lorentz transformation to the laboratory frame gives an electric field component in the $\mathrm{ab}$ planes to be $v_{\mathrm{ff}} \mathrm{B}|\cos \phi|$. Thus the scaling seen in Fig. 2 may not be a sign of strictly independent $2 \mathrm{D}$ pancake vortices, but just weakly-coupled $\mathrm{Cu}-\mathrm{O}$ bilayers. Note that this conclusion is not in conflict with the idea of a 2D-3D crossover in [9].

Implications of the Dip. Having addressed the apparent inconsistencies of the occurrence of the dip in the 2D regime, we now consider the implications of our measurements. Clearly the simple model described above and in [1] does not satisfactorily explain the dip and it seems likely that the effects of vortex-vortex interactions are its most serious deficiency. As a result, these data may be a useful probe of vortex-vortex interactions in systems with well-defined pinning centers.

\section{EFFECTS OF RANDOMNESS}

Our goal here is to consider whether the differences between irradiated and unirradiated samples (e.g., Fig. 2) other than the dip can be quantitatively understood based on the randomness of ion-core defect locations. However, since flux flow dissipation by unpinned vortices is a thermally activated process, data at one temperature alone are not adequate. We therefore have analyzed data similar to Fig. 2 but taken at four temperatures $(75,80,85$ and $90 \mathrm{~K})$, where $T_{c}=102.2$ and $104.2 \mathrm{~K}$ for the irradiated and unirradiated sections of the epitaxial film sample. Such data for rotations in a $6 \mathrm{~T}$ field for the irradiated section only are shown in Fig. 3. The Arrenhius plots at all fields are strictly linear, indicating the activation energies are $\sim 1-T / T_{c}$. This is consistent with

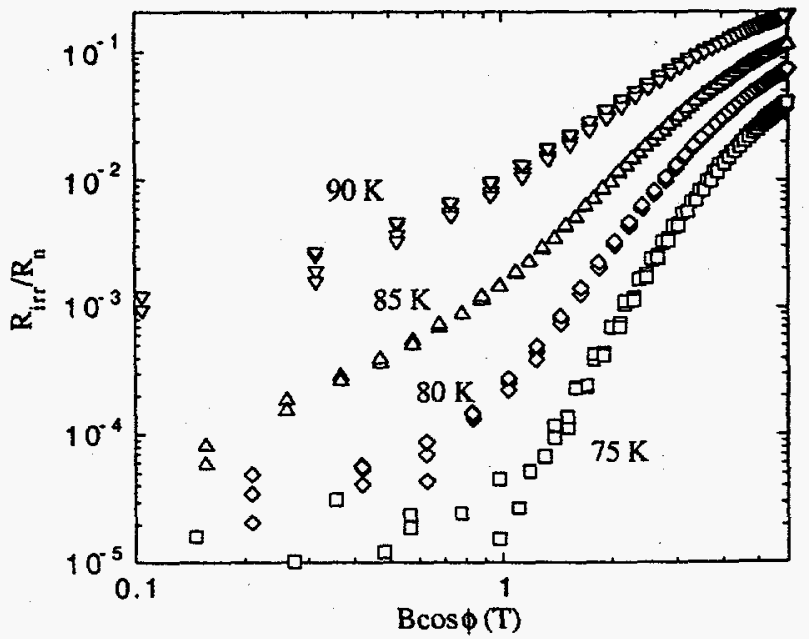

Fig. 3. Effect of temperature on the dissipation as a function of pancake vortex density for a $2 \mathrm{~T}$ irradiation dose. 
vortex-vortex interactions, as well as depinning from the ion cores of radius $r_{\text {core }}>\xi$, the vortex core diameter. For $B|\cos \phi|=0.4 \mathrm{~T}<<\mathrm{B}_{\phi}=2 \mathrm{~T}$, the activation energy is more than four times larger than at $6 \mathrm{~T}$, and we assume this is due to activation out of the ion core pinning sites. We make a simple power-law extrapolation of this low-field behavior to higher fields, subtract it from the total and obtain the dissipation from the 'unpinned' vortices, $R_{u n}$. At the highest fields this extrapolation can be questioned, but then the total dissipation is so large that the error would be small.

We proceed by analyzing the 'unpinned' vortex dissipation according to its Arrenhius behavior:

$$
\frac{R_{u n}}{R_{n}} \sim n_{u n} v \exp \left(E(0)\left(1-T / T_{c}\right) / k T\right)
$$

where $\mathrm{n}_{\mathrm{un}}$ is the number of unpinned vortices, $v$ the attempt frequency and $E(0)$ the activation energy. Extrapolating the Arrenhius plot to $T=T_{c}$, we obtain values of $R_{u n}$ as a function of $B|\cos \phi|$, which should be proportional to $n_{u n}$, if we assume a constant $v$. These are shown as $(+)$ symbols in Fig. 4 as the fraction 'unpinned', $F_{u n} \sim n_{u n} / B / \cos \phi \mid$. With the temperature dependence thus accounted for, we can compare to simple models which address the random defect positions.

We have numerically determined coincidences between random distributions of defects (both 576 and 900 defects are shown) and rigid Abrikosov lattices of various spacings, $a_{0}$, (defined by $B=2 \Phi_{\alpha} / \sqrt{3} a_{0}^{2}$ ), or equivalently, $B / B_{\phi}$. Using the measured ion-core diameter $d_{0}=10 \mathrm{~nm}$ [12], only a very small fraction of the vortices are pinned by cores except for $B / B_{\phi} \ll<1$, in contrast to the experiment. Therefore, we modeled a non-rigid vortex lattice by simply increasing the effective core radius. Good agreement was not possible using a larger, but field-independent, effective core radius, $r_{e f f}$ However, the interaction energy between two vortices separated by $r_{v y}$ is $\sim \ln \left(\lambda / r_{v v}\right)$. As a result, for unperturbed separations of $a_{0}$, the differences in interaction energies, for equal fractional deviations, are independent of $\mathrm{a}_{0}$.

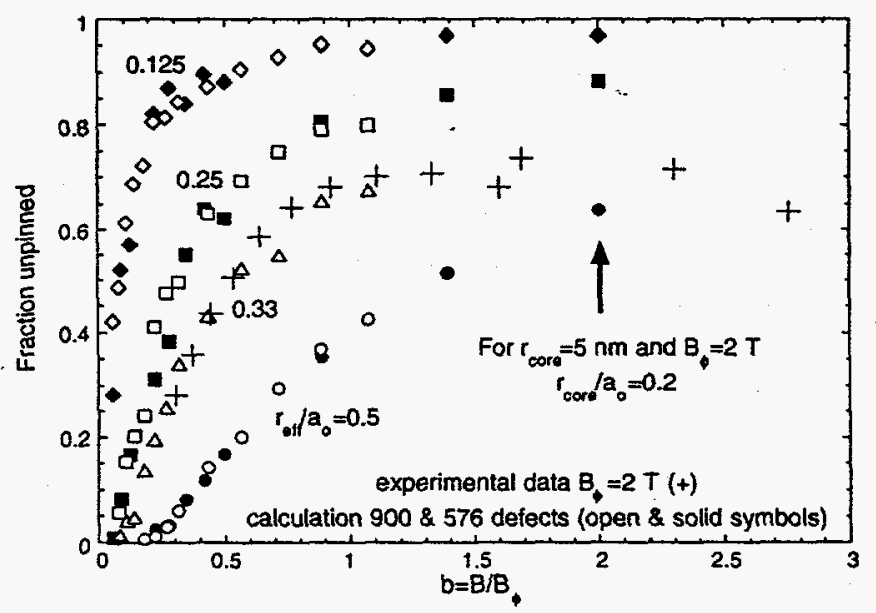

Fig. 4. Comparison of experimental 'unpinned' vortex dissipation with calculations for random ion-core defects.
We can model this by choosing $r_{\text {eff }}$ to be proportional to $a_{0}$, and such calculations are shown in Fig. 4 for various $r_{\text {eff }} / a_{0}$. Note that the experimental data, $F_{u n}$, has been scaled arbitrarily, since the scale factors in Eq. 1 are unknown. The 'best' fit is for $r_{e f f} / a_{0}=0.33$, but since the experimental data at lowest fields depends most sensitively on background subtraction (see Fig. 3), values of $r_{e f f} / a_{o}$ as low as 0.2-0.25 cannot be ruled out. Such large deviations in vortex positions have been observed [14] by scanning tunneling microscopy in ion-irradiated $\mathrm{Bi}_{2} \mathrm{Sr}_{2} \mathrm{CaCu}_{2} \mathrm{O}_{\mathrm{x}}$ and $\mathrm{NbSe}_{2}$, and ultrasound absorption [15] also indicates a softer lattice at smaller fields.

The drop in the experimental data at highest fields can be explained by the finite physical core radius, $r_{\text {core }}=d_{0} / 2$ which should be added to $r_{\text {eff }}$, but which is negligible at low fields.

For $\mathrm{B} / \mathrm{B}_{\phi}>>1$, all ion cores pin a vortex so the unpinned fraction becomes $F_{u n}=1-B_{\phi} / B$. Note that placing two vortices in a defect is energetically unfavorable due the very small ratio of physical core radius, $r_{\text {core, }}$ to penetration length, $\lambda$.

\section{INTERACTIONS WITH PINNED VORTICES}

In order to account for the interactions of unpinned vortices with those in ion-core defects, an effective dose field is introduced. To see this, the data in Fig. 2 are plotted using linear scales in Fig. 5 to emphasize the high field regime, $B>B_{\phi}$. These give the appearance that the primary effect of irradiation is to shift the resistance curves to higher values of the pancake density, $n_{\phi}$. In such an interpretation, the shift in $n_{\phi}$ at constant resistance can be regarded as the number of pancakes firmly pinned by the defects: this can be thought of as the effective dose field, $B_{\dot{\phi}}$, of the columnar defects to be compared with the actual dose field, $B_{\phi}=2 \mathrm{~T}$. Figure 6 shows the temperature dependence of $B_{\phi} / B_{\phi}$, interpreted in this way. These determinations of $B_{\phi} \dot{\phi}$ are complemented by measurements of $\mathrm{J}_{\mathrm{C}}$ at lower temperatures where the linear response regime is inaccessable at available field strengths.

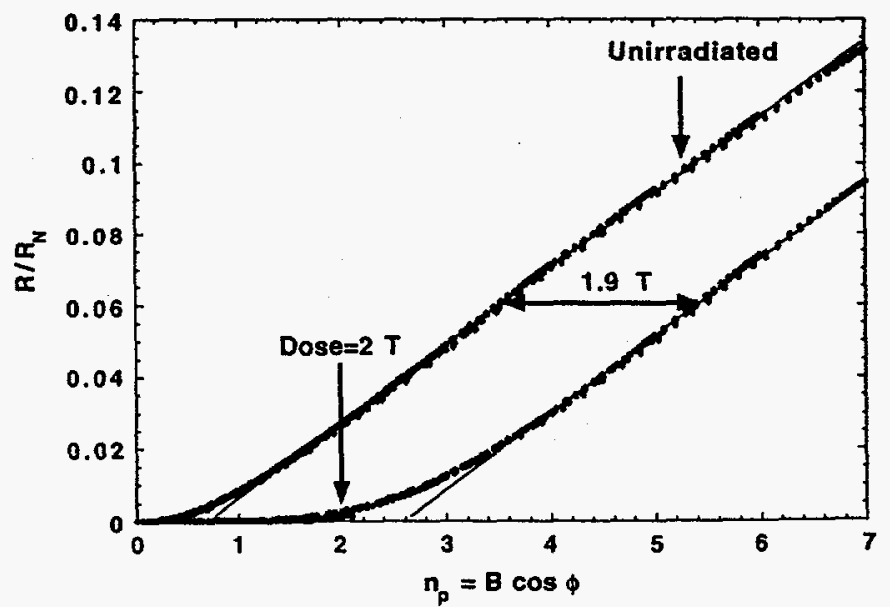

Fig. 5. Data of Fig. 1 plotted on linear scales to show $B_{\dot{\phi}}$. 


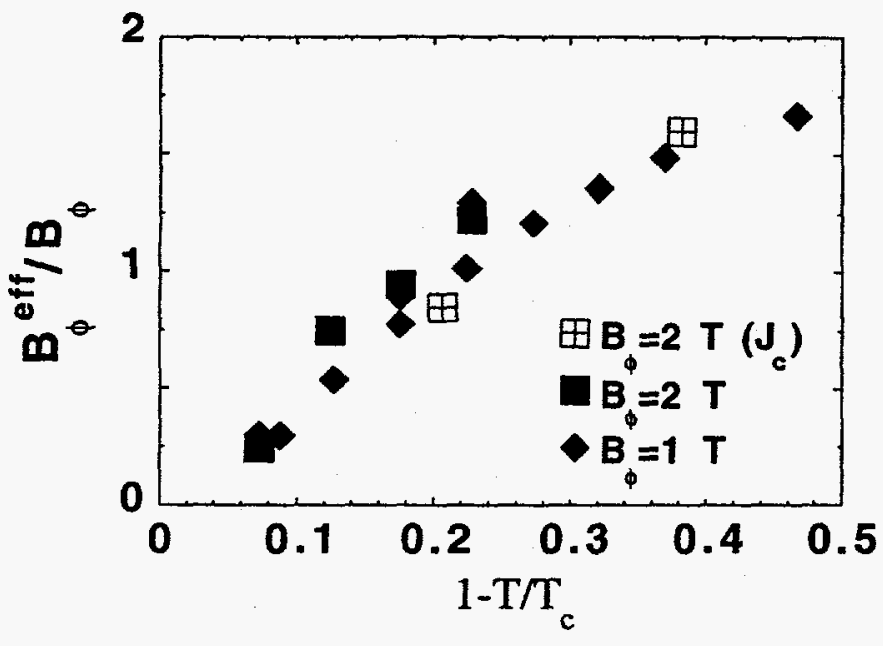

Fig. 6. The effective dose field vs. temperature.

Enhancements of $\mathrm{J}_{\mathrm{C}}$ are found up to at least $3 \mathrm{~T}$ at $60 \mathrm{~K}$ (and 6-7 $\mathrm{T}$ at $40 \mathrm{~K}$ ). There is a fair amount of subjectiveness to the $\mathbf{B}_{\dot{\phi}}$ data: the irradiated and unirradiated data of Fig. 5 are not perfectly parallel (as B approaches $B_{c_{2}}(T)$, they must converge); and the low-temperature data $\left(\mathrm{J}_{\mathrm{C}}\right)$ could not be taken over a sufficiently wide range of currents to define a systematic criterion. Nonetheless, from Fig. 6, we see that $B_{\dot{\phi}}$ is temperature dependent: it vanishes near $T_{c}$ and rises above $B_{\phi}$ at low temperatures. The absence of any apparent feature corresponding to $B=B_{\phi}$ is consistent with our TEM observations of the ion tracks, which is indistinguishable by eye from a simulated random distribution, but which indicates a significant degree of contact (or overlap) of tracks, together with large areas which contain no ion tracks.

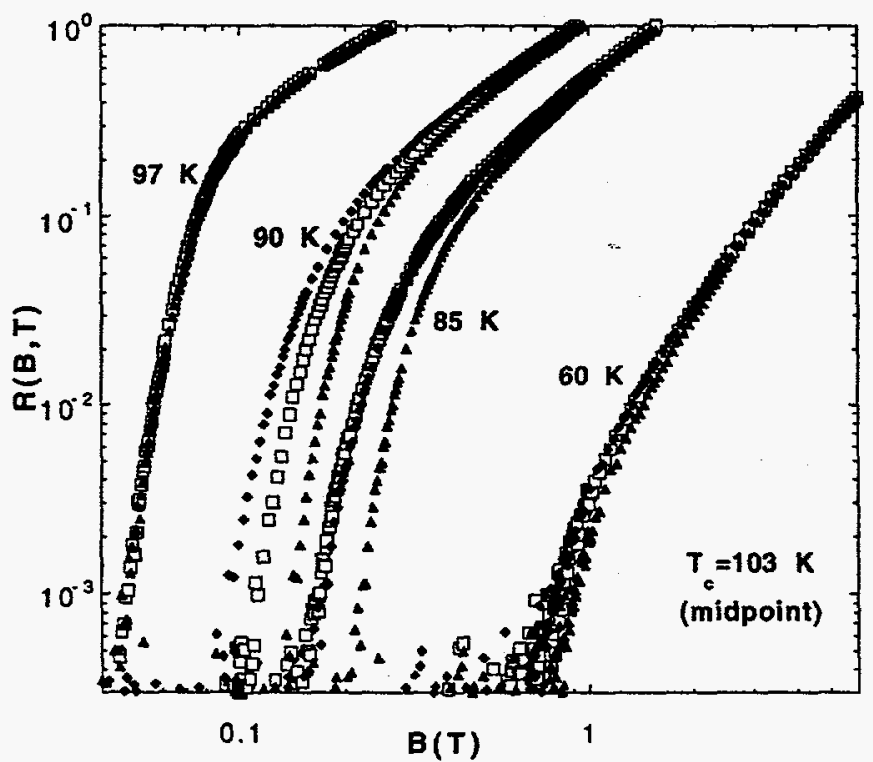

Fig. 7. For $\mathrm{B}_{\phi}=0.05 \mathrm{~T}$ : irradiated (triangles); unirradiated (open squares); irradiated data shifted by $\mathrm{B}_{\phi}$ (diamonds).

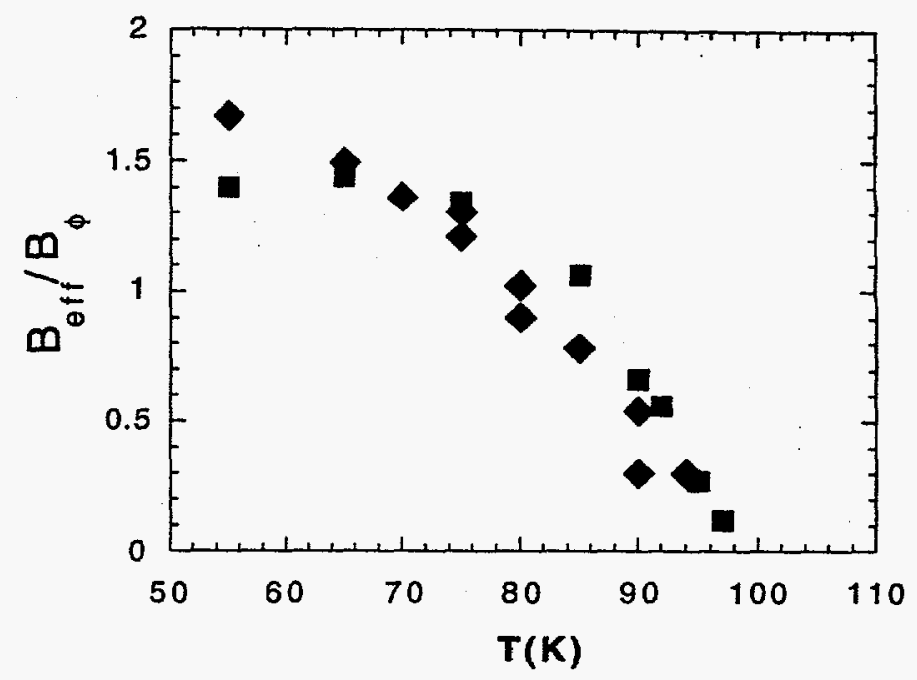

Fig. 8. Comparison of effective dose field for $B_{\phi}=0.05 T$ (squares) and $\mathrm{B}_{\phi}=2 \mathrm{~T}$ (diamonds).

It seems clear that a treatment of vortex-vortex interactions will be necessary to fully understand how the effective dose can be larger than the actual dose of defects. A more rigorous confirmation and a further exploration of this effect requires samples with lower $B_{\phi}$, to obtain larger $\mathrm{B}_{\phi}^{\prime} / \mathrm{B}_{\phi}$ ratios. Data on similar epitaxial $\mathrm{Tl}_{2} \mathrm{Ba}_{2} \mathrm{CaCu}_{2} \mathrm{O}_{y}$ films irradiated to a dose of $0.05 \mathrm{~T}$ are shown in Fig. 7. At $85 \mathrm{~K}$, there is no difference if the irradiated curve is shifted by $0.05 \mathrm{~T}$, which is consistent with all defects being occupied by vortices. However, at $90 \mathrm{~K}$ such a shift overestimates the differences indicating a smaller effective field and at $97 \mathrm{~K}$ there is virtually no difference after irradiation, i.e., $B_{\dot{\phi}}=0$. Finally, at $60 \mathrm{~K}$ a greater shift than $0.05 \mathrm{~T}$ is required to match the data. Qualitatively this is the same result found for the film with a $2 \mathrm{~T}$ dose, and Fig. 8 demonstrates the nearly equivalent behavior, indicating that this general trend may be universal.

\section{SUMMARY}

We have made careful measurements in a magnetic field of the resistive dissipation in high-quality epitaxial films of $\mathrm{Tl}_{2} \mathrm{Ba}_{2} \mathrm{CaCu}_{2} \mathrm{O}_{\mathrm{x}}$, both in the pristine state and after ion irradiation to various doses. These data were collected as a function of the field angle with respect to the crystallographic c-axis (which coincided in most samples with the ion-track direction) over a wide range of fields and temperatures. The appearance of a dip in dissipation for fields aligned with the columnar defects of the irradiated sample was surprising since such a dip has been taken as evidence for 3D vortices yet our data is collected at fields and temperatures where the thermal activation [1] model and scaling [9] measurements imply that the vortices exhibit strongly $2 \mathrm{D}$ behavior. We have presented a qualitative model to address the apparent contradiction with thermal activation, but recognize that important effects, such as vortex interactions, are missing and may be the key to a 
quantitative reconciliation. We also suggest that the scaling behavior may be connected with weak interlayer coupling and not necessarily with the occurrence of strictly $2 \mathrm{D}$ vortices.

We have shown that the randomness of the defect positions washes out any sharp distinction between fields less than or greater than the dose field. Comparing data with a simple model of randomness, one concludes that to maximize the occupation of favorable ion-core pinning sites, the vortex lattice will distort by a reasonable fraction $(\sim 0.25-0.33)$ of its intervortex spacing, $a_{0}$.

A second result of vortex-vortex interactions, is the appearance of an effective dose field, $B_{\dot{\phi}}$, which is temperature dependent. Because of interactions between the 'unpinned' vortices and those in ion-core defects, $B_{\phi}$ ' rises to many times $\mathrm{B}_{\phi}$ for $\mathrm{T}<<\mathrm{T}_{\mathrm{c}}$.

In total, these experiments indicate that including vortexvortex interactions in important to a full understanding of flux dynamics in high-temperature superconductors. The picture is summarized by Fig. 4, in which there is presumably one universal curve for the $r_{\text {eff }} / a_{0}$ value corresponding to the appropriate effect of vortex interactions on the rigidity of the vortex lattice. In principle, the vortex interactions with pinned vortices and thermal effects represented in Fig. 6 and 8 should rescale the dose field used in Fig. 4, but the experimental evaluation of the 'unpinned' fraction is non trivial since the data for Fig. 4 was determined from activation analyses over a range of temperatures.

Further work should be done on other high-temperature superconducting materials and other doses to understand the connection between these results. Among other things, it would be useful to verify the universality of the value of $r_{\text {eff }} / a_{0}$ and of the temperature dependence of the effective dose field. In principle, the ideas here can be extended to other types of defects, e.g., point defects in materials with $3 D$ vortices, like $\mathrm{YBa}_{2} \mathrm{Cu}_{3} \mathrm{O}_{7}$.

\section{REFERENCES}

[1] D.H. Kim, K.E. Gray, R.T. Kampwirth, J.C. Smith, D.S. Richeson, T.J. Marks, J.H. Kang, J. Talvacchio and M. Eddy, Physica C177, 431 (1991).

[2] S. Rye, S. Doniach, G. Deutscher and A. Kapitulnik, Phys. Rev. Lett. 68, $710(1992)$

[3] C. Duran, J. Yazyi, F. de la Cruz, D.J. Bishop, D.B. Mitzi and A. Kapitulnik, Phys. Rev. B44, 7737 (1991).

[4] L. Civale, A.D. Marwick, T.K. Worthington, M.A. Kirk, J.R. Thompson, L. Krusin-Elbaum, Y. Sun, J.R. Clem and F.H. Holtzberg, Phys. Rev. Lett. 67, 648 (1991).

[5] J.R. Thompson, Y.R. Sun, H.R. Kerchner, D.K. Christen, B.C. Sales, B.C. Chakoumakos, A.D. Marwick, L. Civale and J.O. Thomson, Appl. Phys. Lett. 60, 2306 (1992); W. Gerhäuser, G. Ries, H.W. Neumüller, W. Schmidt, O. Eibl, G. Saemann-Ischenko and S. Klaumünzer, Phys. Rev. Lett. 68, 879 (1992).

[6] L. Klein, E.R. Yacoby, Y. Yeshurun, M. Konczykowski and K Kishio, Phys. Rev. B48, 3523 (1993); and Physica A200, 413 (1993).

[7] P. Kummeth, C. Struller, H.W. Neumulller and G. Saemann-Ischenko, Appl. Phys. Lett. 65, 1302 (1994).

[8] R.C. Budhani, W.L. Holstein and M. Suenaga, Phys. Rev. Lett. 72, 566 (1994).

[9] P.H. Kes, J. Aarts, V.M. Vinokur and C.J. van der Beek, Phys. Rev. Lett. 64, 1063 (1990).

[10] This suggestion stems from the reasonable expectation of significant film strain caused by the deposited heavy ions expanding the substrate.

[11] K.E. Gray, Appl. Supercond. 2, 295 (1994).

[12] K. E. Gray, J. D. Hettinger, D. J. Miller, B. R. Washburn C. Moreau, C. Lee, B.G. Glagola and M.M. Eddy, Phys. Rev. B54, 0000 (August 1, 1996).

[13] The force is perhaps more correctly thought of as the magnetic pressure due to the flux gradient caused by the transport current density, see P.W. Anderson and Y.B. Kim, Rev. Mod. Phys. 36, 39 (1964).

[14] S. Behler, S.H. Pan, P. Jess, A. Baratoff, H.J. Güntherodt, F. Levy, G. Wirth and J. Wiesner, Phys. Rev. Lett. 72, 1750 (1994), U.C. Täuber, H. Dai, D.R. Nelson and C.M. Lieber, Phys. Rev. Lett. 74, 5132 (1995).

[15] C. Hucho and M. Levy, Phys. Rev. Lett. 77, 1370 (1996). 